Case Report

\title{
Pigmented Villonodular Synovitis Causing Osteonecrosis of the Femoral Head: A Case Report
}

\author{
Tomohiro Mimura, Taku Kawasaki, Keitaro Yagi, Kanji Mori, \\ Shinji Imai, and Yoshitaka Matsusue \\ Department of Orthopaedic Surgery, Shiga University of Medical Science, Tsukinowa-cho, Seta, Otsu, Shiga 520-2192, Japan \\ Correspondence should be addressed to Tomohiro Mimura; tmimura@belle.shiga-med.ac.jp
}

Received 7 November 2013; Accepted 1 December 2013

Academic Editors: P. Carpintero, C. C. Liao, and B. Unver

Copyright (c) 2013 Tomohiro Mimura et al. This is an open access article distributed under the Creative Commons Attribution License, which permits unrestricted use, distribution, and reproduction in any medium, provided the original work is properly cited.

We report a case of a 27-year-old man with pigmented villonodular synovitis of the hip joint with coincident osteonecrosis of the femoral head. According to our review of the English-language literature, no detailed report of osteonecrosis of the femoral head complicated with pigmented villonodular synovitis has been published. Preoperative X-ray images showed joint narrowing and severe multiple bone erosions at the acetabulum and femoral neck. Magnetic resonance imaging revealed a lowintensity band attributable to osteonecrosis of the femoral head and massive diffuse pigmented villonodular synovitis lesions. Comparison of a three-dimensional computed tomographic image of this patient with an angiographic image of a normal individual demonstrated proximity of the pigmented villonodular synovitis-induced bone erosions to the medial and lateral femoral circumflex arteries and retinacular arteries, suggesting likely the compromise of the latter by the former. We propose that the massive pigmented villonodular synovitis may have contributed to the pathogenesis of osteonecrosis of the femoral head in this patient. We performed open synovectomy and total hip arthroplasty. No operative complications occurred, and no recurrence of the pigmented villonodular synovitis was detected for 3 years after the operation.

\section{Introduction}

Pigmented villonodular synovitis (PVNS) was named and described by Jaffe et al. in 1941 [1]. However, the first description of PVNS is commonly attributed to Chassaignac in 1852. Myers and Masi estimated that the overall prevalence of PVNS is 1.8 per million people [2]. They reported that the knee is the most commonly affected joint, accounting for up to $80 \%$ of cases, and that the hip is the second most commonly affected joint, accounting for $15 \%$. In contrast, osteonecrosis of the femoral head (ONFH) is a relatively common disease, first described by Heimann and Freiberger in 1960 [3]. We herein report a case of ONFH complicated with PVNS, which, to our knowledge, is the first detailed report of this presentation in the English-language literature. We propose that the massive PVNS may have contributed to the occurrence of ONFH in this patient, creating multiple bone erosions around the femoral neck.

\section{Case Presentation}

The patient was a 27 -year-old man (weight: $66 \mathrm{~kg}$; height; $166 \mathrm{~cm}$ ) with no underlying disease. This patient became aware of coxalgia 1 year earlier. The coxalgia was associated with continuous pain that became more severe, and at the time of presentation he could not walk without an axillary crutch. X-ray images showed joint narrowing and multiple bone erosions of the left hip joint (Figure 1). Computed tomography (CT) clearly showed femoral neck and acetabular bone erosions as well as ONFH (Figures 2(a) and 2(b)). In contrast, magnetic resonance (MR) imaging showed ONFH and mass lesions around the femoral neck. T2-weighted fatsuppressed MR imaging also revealed an irregular tumoral lesion (Figure 2(c)). The patient had type C ONFH according to the Nishii et al. classification [4] and stage $4 \mathrm{ONFH}$ according to the Association Research Circulation Osseous classification [5]. 


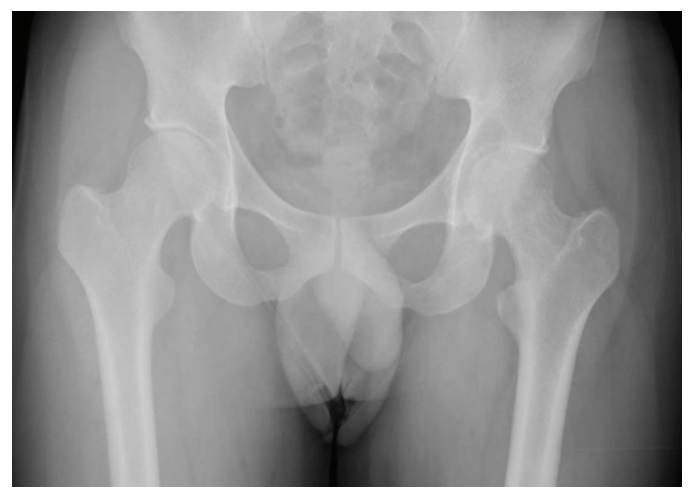

(a)

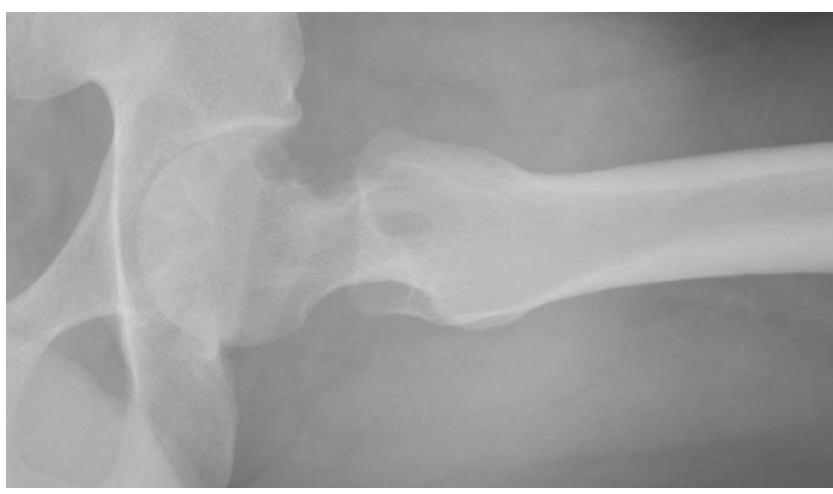

(b)

FIGURE 1: (a, b) Joint narrowing and multiple bone erosions of the hip joint are seen on this roentgenogram.

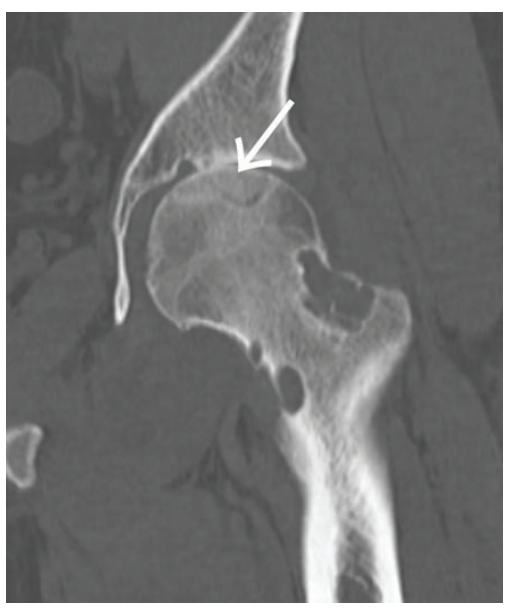

(a)

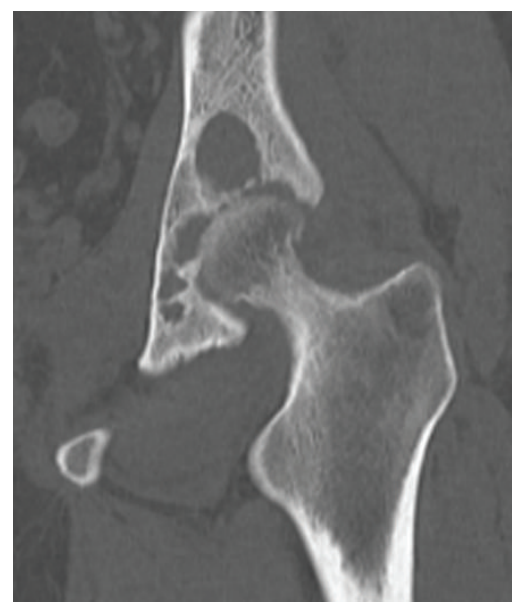

(b)

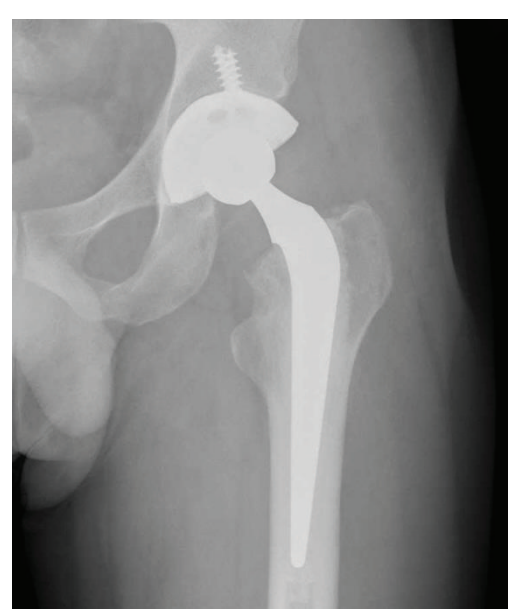

(d)

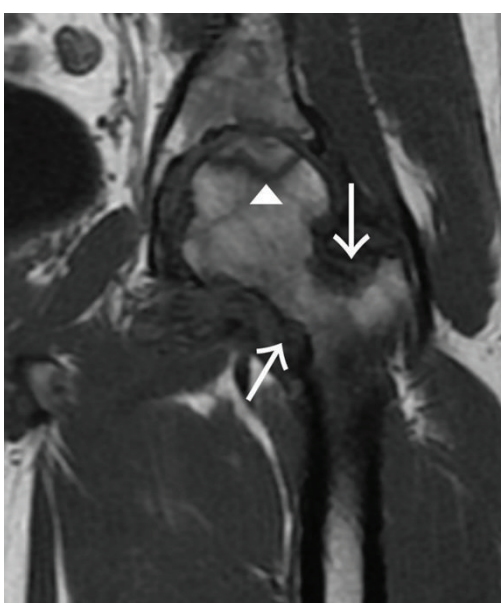

(c)

Figure 2: (a, b) Computed tomography image showing the femoral neck and acetabular bone erosions, and area of bone necrosis (arrow). (c) Magnetic resonance image showing a typical low-intensity band of osteonecrosis of the femoral head (arrowhead) and mass lesions (arrows) around the femoral neck on T1-weighted imaging. (d) The patient underwent total hip arthroplasty with complete synovectomy and tumor resection using an argon laser. 


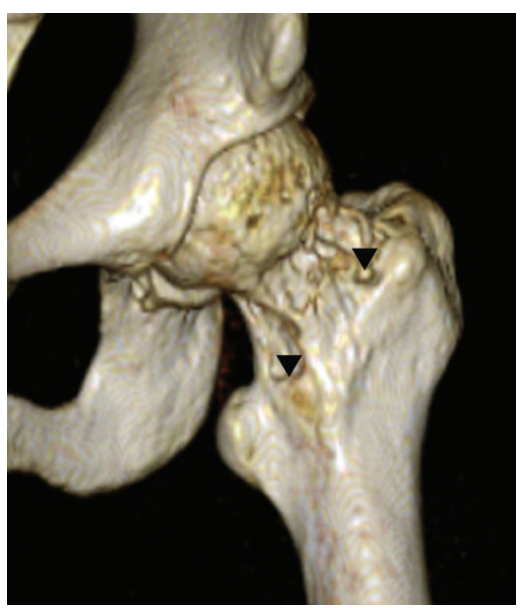

(a)

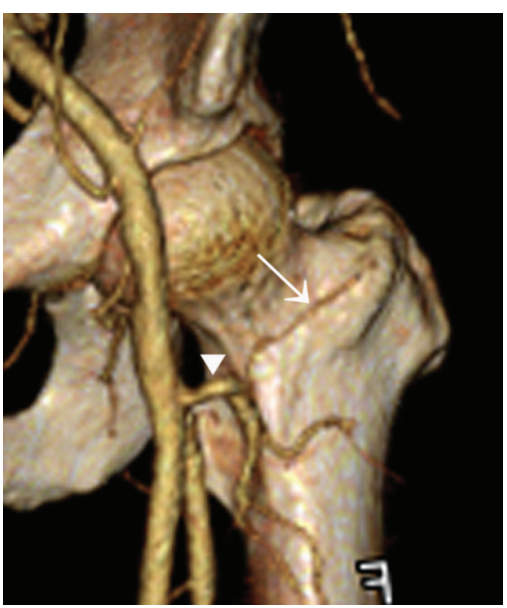

(b)

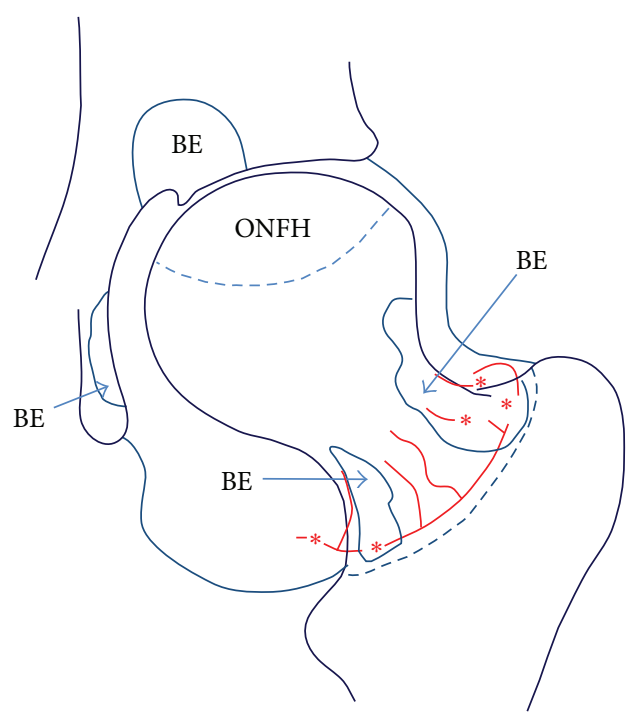

(c)

Figure 3: (a) Reconstructed three-dimensional computed tomography image shows bone erosions of the femoral head-neck junction (arrowhead). (b) Reconstructed three-dimensional computed tomography-angiography image of a normal individual. The lateral femoral circumflex artery (arrow) and base of medial femoral circumflex artery (arrowhead) are visible. (c) BE: bone erosion; ONFH: osteonecrosis of the femoral head. A schema of the BE area and ONFH. Asterisks show a break or compression of the lateral femoral circumflex artery, base of the medial femoral circumflex artery, and/or retinacular arteries. These arteries were considered to be injured by pigmented villonodular synovitis, creating multiple BE around the femoral head-neck junction.

We performed an arthroscopic biopsy before radical treatment. Histological analysis of the synovial tissue showed diffuse PVNS. The patient subsequently underwent total hip arthroplasty (THA) (KYOCERA Medical Corporation, Osaka, Japan) with complete synovectomy and tumor resection using an argon laser (Figure 2(d)). Severe invasive PVNS was seen in the inferior space of the hip joint, particularly around the femoral neck.

Pathological findings from an analysis of permanent preparations of the synovium and femoral head were consistent with PVNS and avascular bone necrosis, respectively. Proliferative histiocyte-like mononuclear cells were observed with multinucleated giant cells in the fibrotic stroma. Hemosiderin-laden macrophages and foamy cells were also occasionally observed. The subchondral zone of the femoral head showed avascular bone necrosis. Both empty lacunae and fat necrosis were found in the resected femoral head. Fibrosis and proliferation of vessels were observed around the necrotic trabeculae, and new bone apposition was present.

After the procedure, the Harris hip score improved from 79 to 97 . No operative complications occurred, and no recurrence of PVNS was detected for 3 years after the procedure.

\section{Discussion}

PVNS is a rare disease and is typically present in young adults between 20 and 40 years of age. PVNS usually affects one joint; involvement of more than one joint is extremely rare [6]. In their paper, Cotton et al. reported that both hips were affected in only 2 of 58 patients [7]. Several previous studies have reported that PVNS is slightly more prevalent in female patients [8]. In the hip joint, the diffuse type is more common than the local type [9]. The predominant roentgenogram findings of PVNS in the hip joint are multiple cyst-like lesions or erosive areas of non-weight-bearing regions of the acetabulum and femoral head and/or femoral neck. Cotton et al. showed the presence of these bony lesions in $95 \%$ of their cases (cortical erosion, 43\%; cyst-like erosion, 90\%) [7].

The etiology of PVNS is unclear. Many theories have been put forward to explain the pathogenesis of PVNS, including etiopathological mechanisms involving inflammatory reactions [1], abnormal cellular and humoral immunity, genetic predisposition, or recurrent hemorrhage following trauma. However, a neoplastic origin currently seems to be the most influential theory [10]. Several reports have described the development of local recurrence and metastasis of PVNS [11]. At present, adjuvant radiation therapy is used to treat aggressive PVNS [11]. However, complete synovectomy and arthroplasty have been used as alternative treatments of PVNS. In previous studies, outcomes have been better after synovectomy with THA than after synovectomy alone $[12,13]$. Yoo et al. reported no recurrent PVNS in their eight cases treated with cementless THA during an average followup of 8.9 years [14]. They concluded that THA with synovectomy is an adequate therapeutic choice for patients with PVNS demonstrating end-stage joint destruction and appears to effectively improve clinical results and prevent disease recurrence.

Atraumatic ONFH progresses to the collapse of the femoral head in many patients and requires surgery to relieve 
hip pain. A systematic review in 2010 found that the causes of ONFH were corticosteroids (11\%, 31/282), excessive alcohol consumption (16\%), idiopathic disease $(11 \%)$, systemic lupus erythematosus $(21 \%)$, sickle cell disease $(14 \%)$, renal failure and/or transplantation (17\%), and human immunodeficiency virus (10\%) [15]. According to our review of the Englishlanguage literature, no case reports of coincident ONFH and PVNS have been reported. We propose that the massive PVNS creating circular bone erosions around the femoral neck may have contributed to the pathogenesis of $\mathrm{ONFH}$ in the present case. Comparison of the three-dimensional (3D) CT image of the femoral neck lesion of this patient (Figure 3(a)) with a 3D CT angiographic image of a normal individual (Figure 3(b)) demonstrates the proximity of the bone erosion to the base of the medial and lateral femoral circumflex arteries. Schematic superimposition of the bone erosions and circumflex arteries (Figure 3(c)) suggests likely the compromise of the latter by the former.

In conclusion, we presented a detailed report of a case of ONFH that appeared to be secondary to PVNS. No recurrence of PVNS was detected for 3 years after the procedure.

\section{Conflict of Interests}

The authors declare no conflict of interests.

\section{References}

[1] H. L. Jaffe, L. Lichtenstein, and C. J. Sutro, "Pingented villonodular synovitis and bursal equivalents of tenosynovial lesion commonly denoted as xanthoma, xanthogranuloma, gaiant cell tumor or myeloplaxoma of tendon sheath, with some considaration of this tendon sheath lesion itself," Archives of Pathology, vol. 31, pp. 731-765, 1941.

[2] B. W. Myers and A. T. Masi, "Pigmented villonodular synovitis and tenosynovitis: a clinical epidemiologic study of 166 cases and literature review," Medicine, vol. 59, no. 3, pp. 223-238, 1980.

[3] W. G. Heimann and R. H. Freiberger, "Avascular necrosis of the femoral head and humeral heads after high-dosage corticosteroid therapy," The New England Journal of Medicine, vol. 263, pp. 672-675, 1960.

[4] T. Nishii, N. Sugano, K. Ohzono, T. Sakai, K. Haraguchi, and H. Yoshikawa, "Progression and cessation of collapse in osteonecrosis of the femoral head," Clinical Orthopaedics and Related Research, no. 400, pp. 149-157, 2002.

[5] J. W. M. Gardeniers, "A new international classification of osteonecrosis of the ARCO Committee on Terminology and Classification," Journal of the Japanese Orthopaedic Association, vol. 66, no. 2, pp. 18-19, 1992.

[6] P. D. Byers, R. E. Cotton, O. W. Deacon et al., "The diagnosis and treatment of pigmented villonodular synovitis," Journal of Bone and Joint Surgery B, vol. 50, no. 2, pp. 290-305, 1968.

[7] A. Cotten, R.-M. Flipo, P. Chastanet, M.-C. Desvigne-Noulet, B. Duquesnoy, and B. Delcambre, "Pigmented villonodular synovitis of the hip: review of radiographic features in 58 patients," Skeletal Radiology, vol. 24, no. 1, pp. 1-6, 1995.
[8] R. H. Dorwart, H. K. Genant, W. H. Johnston, and J. M. Morris, "Pigmented villonodular synovitis of synovial joints: clinical, pathologic, and radiologic features," American Journal of Roentgenology, vol. 143, no. 4, pp. 877-885, 1984.

[9] P. Aglietti, G. V. Di Muria, E. A. Salvate, and G. Stringa, "Pigmented villonodular synovitis of the hip joint. (Review of the literature and report of personal case material)," Italian Journal of Orthopaedics and Traumatology, vol. 9, no. 4, pp. 487496, 1983.

[10] A. S. Rao and V. J. Vigorita, "Pigmented villonodular synovitis (giant-cell tumor of the tendon sheath and synovial membrane). A review of eighty-one cases," Journal of Bone and Joint Surgery A, vol. 66, no. 1, pp. 76-94, 1984.

[11] L. M. Li and J. Jeffery, "Exceptionally aggressive pigmented villonodular synovitis of the hip unresponsive to radiotherapy," Journal of Bone and Joint Surgery B, vol. 93, no. 7, pp. 995-997, 2011.

[12] L. Vastel, P. Lambert, G. De Pinieux, O. Charrois, M. Kerboull, and J.-P. Courpied, "Surgical treatment of pigmented villonodular synovitis of the hip," Journal of Bone and Joint Surgery A, vol. 87, no. 5, pp. 1019-1024, 2005.

[13] A. G. Della Valle, F. Piccaluga, H. G. Potter, E. A. Salvati, and R. Pusso, "Pigmented villonodular synovitis of the hip: 2to 23-year followup study," Clinical Orthopaedics and Related Research, no. 388, pp. 187-199, 2001.

[14] J. J. Yoo, Y. S. Kwon, K.-H. Koo, K. S. Yoon, B. W. Min, and H. J. Kim, "Cementless total hip arthroplasty performed in patients with pigmented villonodular synovitis," Journal of Arthroplasty, vol. 25, no. 4, pp. 552-557, 2010.

[15] M. A. Mont, M. G. Zywiel, D. R. Marker, M. S. McGrath, and R. E. Delanois, "The natural history of untreated asymptomatic osteonecrosis of the femoral head: a systematic literature review," Journal of Bone and Joint Surgery A, vol. 92, no. 12, pp. 2165-2170, 2010. 


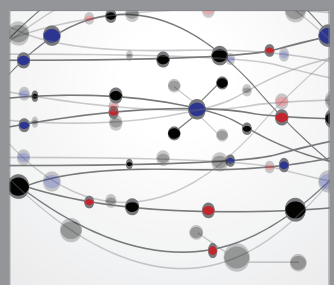

The Scientific World Journal
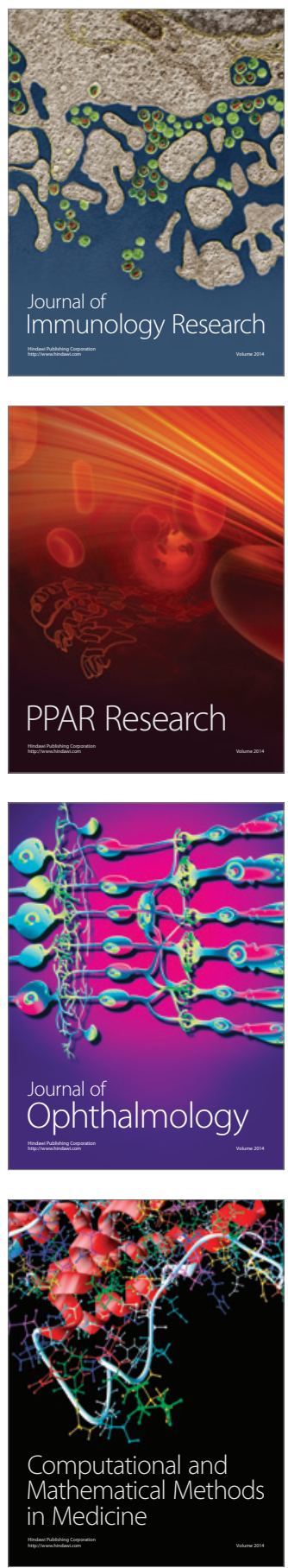

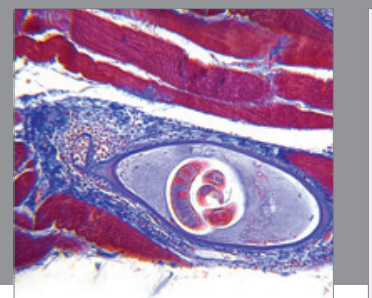

Gastroenterology

Research and Practice
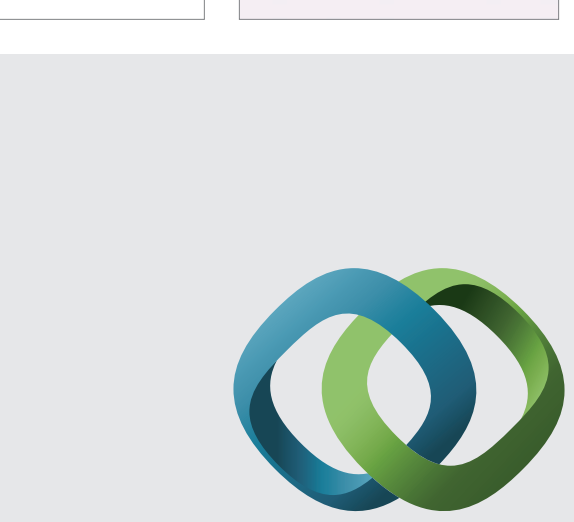

\section{Hindawi}

Submit your manuscripts at

http://www.hindawi.com
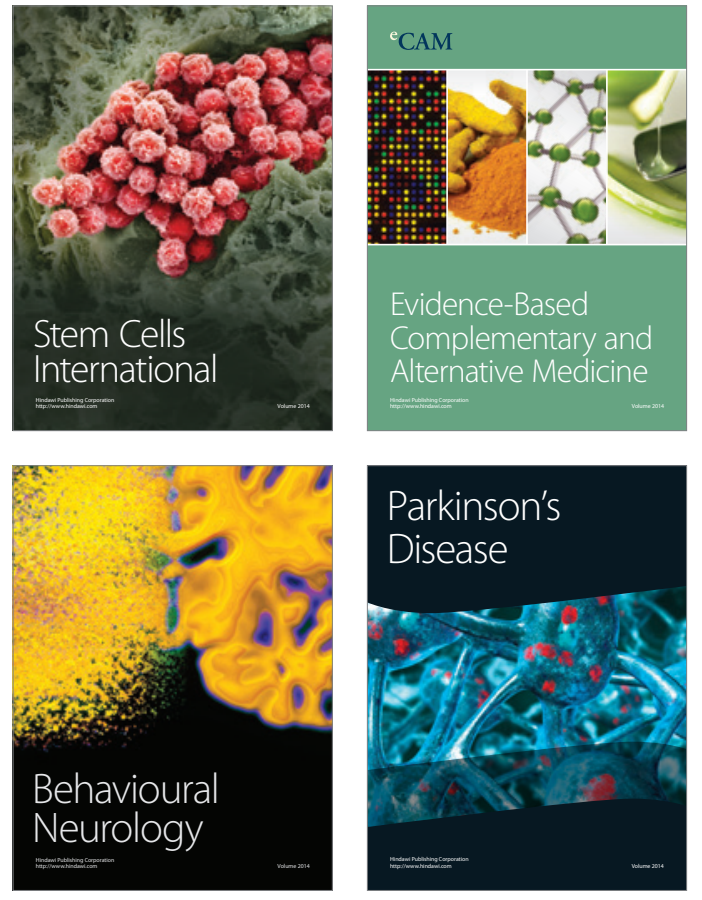
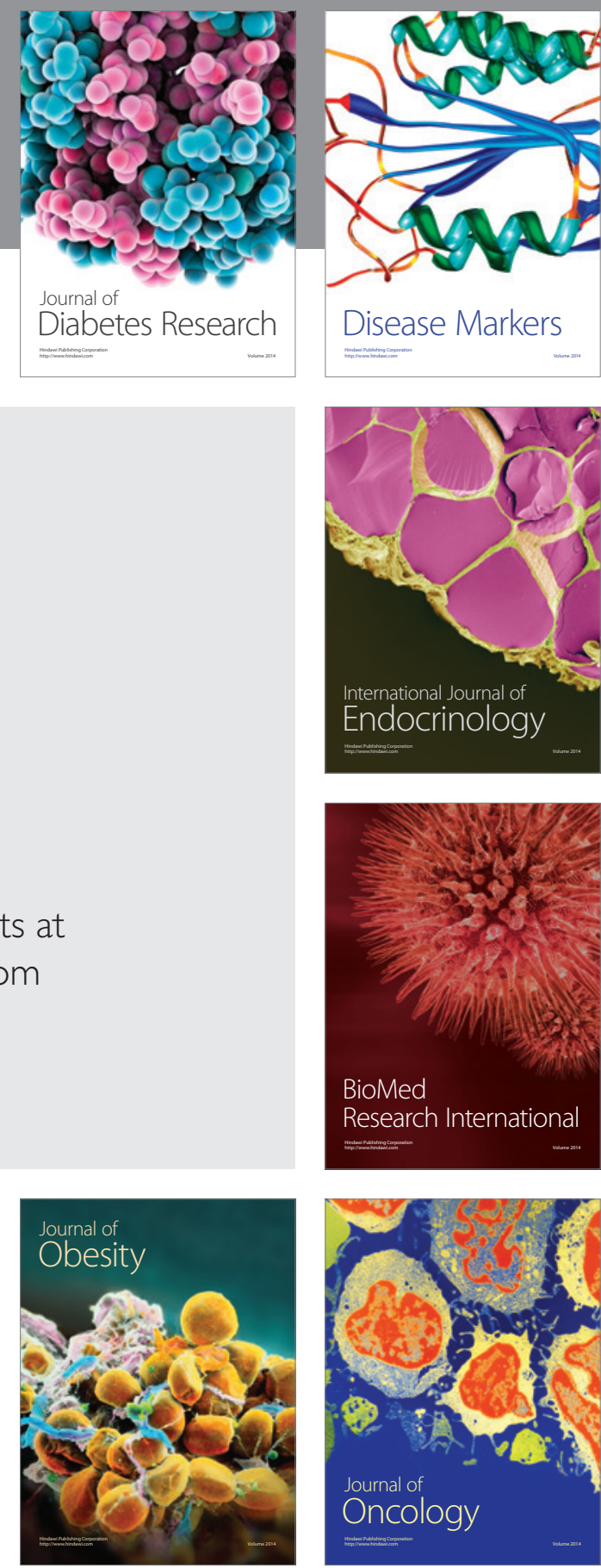

Disease Markers
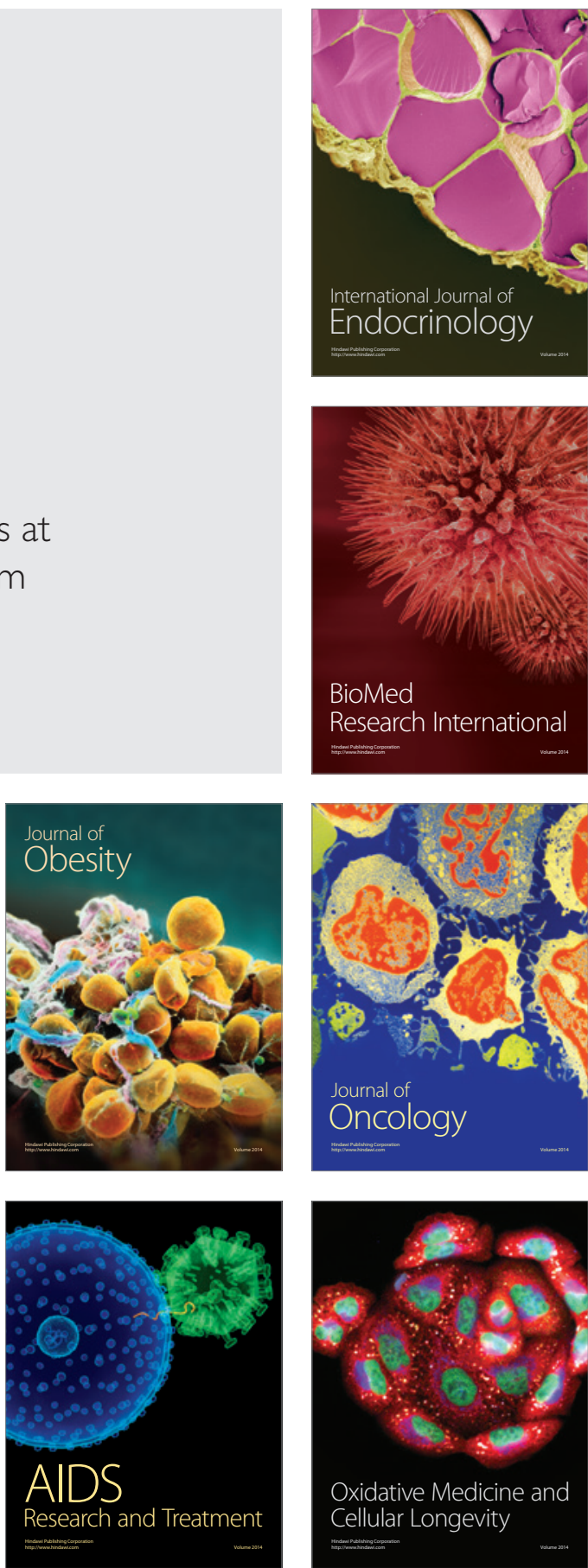